\title{
Laparoscopic Diagnosis and Treatment of Stage 4 Endometriosis after Traumatic Agni karma with Abdominal Scars: a Case Report.
}

Feras Sendy ${ }^{1}$, Wd Sendy ${ }^{2}$, Sameer Sendy ${ }^{3}$

${ }^{1}$ Obstetrics and Gynecology Department, King Fahad Medical City, Riyadh, SaudiArabia.

${ }^{2}$ King Abdullah University Hospital, Princess Nourah Bint Abdulrahman University, Riyadh, Saudi Arabia.

${ }^{3}$ King Saud University, KSA

*Corresponding Author: Feras Sendy. 1Obstetrics and Gynecology Department, King Fahad Medical City, Riyadh, SaudiArabia. Received Date: April 20, 2019; Accepted Date: May 28, 2020; Published Date: June 11, 2020.

Citation: Sendy F, Sendy W, Sendy S. (2020) Laparoscopic Diagnosis and Treatment of Stage 4 endometriosis After Traumatic Agni karma with Abdominal Scars: a Case Report.: Observational study. Obstetrics Genecology and Reproductive Sciences, 4(1):DOI:10.31579/2578-8965/040

Copyright: () 2020. Feras Sendy. This is an open-access article distributed under the terms of the Creative Commons Attribution License, which permits unrestricted use, distribution, and reproduction in any medium, provided the original author and source are credited.

\begin{abstract}
Agni karma refers to the use of heated metals by traditional physicians. Endometriosis is defined as implantation of endometrial cells outside the endometrial cavity. Presenting symptoms are dyspareunia and dysmenorrhea. Recognition and awareness of such disorder are vital to avoid skin damage. A 31 years old nulliparous presented with dysmenorrhea and dyspareunia after unsuccessful attempts to alleviate symptoms by heated metals. Stage 4 endometrioses was diagnosed via laparoscopy and bilateral ovarian cystectomy was done for endometriomas. Agni karma is an unacceptable treatment for endometriosis as it results in avoidable body damage. Using heated rods is contraindicated in endometriosis, as it does neither alleviate symptoms nor treat the condition. It is used due to its lower cost, rapidity to treat the illness and non-complex equipment. To prevent unnecessary body damages, awareness is crucial along with consulting legal healthcare centers where medical and surgical treatment from qualified healthcare professionals is provided.
\end{abstract}

Keywords: endometriosis; agni karma; laparoscopy; dysmenorrhea and dyspareunia

\section{Introduction}

Endometriosis is a painful gynecological disorder, which occurs when tissues that resemble the normal endometrium grows outside the uterus(1). The disease affects several parts of the female reproductive system, including ovaries, fallopian tubes, and the pelvic lining. Sometimes the disease may spread to organs outside. In severe cases, the endometrial tissue swells and breaks resulting in bleeding(1). This is usually accompanied by severe pain. To stop the progress of endometriosis, immediate treatment is required. Although medical treatment can be used to relieve pain and arrest bleeding, getting rid of the endometrial tissues requires invasive procedures such as laparoscopy. However, in some parts of the world such as Saudi Arabia, Yemen and India, therapeutic heat burns, which involve heating the symptomatic site with a hot metal are used to treat such diseases. According to the current medical guidelines, such procedures are unacceptable, and they should not be used in treatment. As such, this paper presents the first case report of a patient with endometriosis that experienced traumatic effects of therapeutic heat burns that ultimately led to seeking medical advice for diagnosis and treatment. The aims to discuss the reasons why therapeutic heat burns are unacceptable and increase awareness in order to diagnose patients with endometriosis.

\section{Case presentation}

A 31 years old nulliparous presented to our hospital with severe dysmenorrhea and dyspareunia for one year. The patient has a normal regular menstrual cycle with no inter-menstrual or post-coital bleeding. She has used multiple herbal treatments that failed to alleviate symptoms. Lastly, she went to a traditional public physician that used heated rods to cauterize her abdomen around a year ago. Figure 1 shows the traumatic effects of the patients' skin due to the use of heated metals without alleviating symptoms.

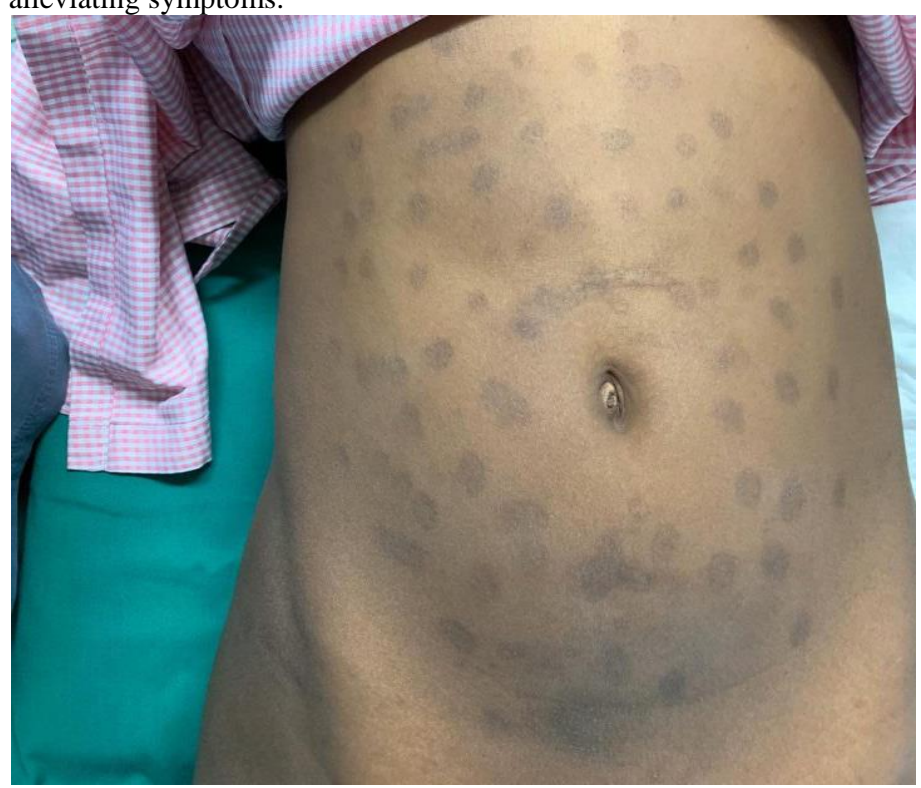

Figure 1: Abdominal scarring from Agni karma.

Sexual activity was impaired with the husband due to dyspareunia and multiple abdominal scars from the heated metals although, she had a pregnancy desire. On examination, the abdomen was soft and lax, with no apparent masses. Her Hemoglobin level was 11.6g/dl, CA 125 was 
$51.3 \mathrm{U} / \mathrm{mL}$ and the blood group was $\mathrm{O}$ negative. Ultrasound assessment showed an anteverted uterus $3.8 \times 3.8 \times 4.2 \mathrm{~cm}$ with multiple uterine fibroids including: posterior intramural fibroid measuring $2.3 \times 2 \mathrm{~cm}$, anterior intramural fibroids $2.7 \times 2.2 \mathrm{~cm}$ and $1.4 \times 1.1 \mathrm{~cm}$ and right lateral intramural fibroids $1.7 \times 1.7 \mathrm{~cm}$ and $1.0 \times 0.9 \mathrm{~cm}$. The endometrial thickness was 0.5. The right and left ovaries had unilocular hypoechoic cyst suggestive of endometrioma measuring $4.1 \times 3.1 \mathrm{~cm}$ and $5.1 \times 3.5$ $\mathrm{cm}$, respectively. The patient was informed and consented for bilateral laparoscopic ovarian cystectomy. With Laparoscopy, we found a stage four endometriosis according to the American fertility staging and laparoscopic bilateral cystectomy was done without complications. Histopathology reports confirmed the diagnosis of endometriomas without malignancy. The patient was discharged on the same day, as the postoperative course was unremarkable. She was prescribed Dienogest (Visanne (®) after explanation in order to have medical and surgical benefits. Follow up appointment showed improvement in symptomatology with no dyspareunia.

\section{Discussion}

According to Nagnath(2), therapeutic heat burns, commonly referred to as "Agni karma" in India is of much importance when applied carefully in healing wounds and other diseases involving tissues. First of all, the chances of recurrence of the disease are low when this method is used. The method involves heating of the bleeding site with a hot metal, which causes coagulation resulting in total closure of bleeding vessels. Secondly, Agni karma ensures that every ill tissue is eradicated from the bleeding site. This removes all disease-causing microorganisms from the site reducing chances of reoccurrence. Lastly, Nagnath (2) claims that the therapeutic heat method is cheaper compared to other surgical methods. The procedure does not require complex equipment like other normal procedures provided a specialist is summoned. It can be carried out at home when medical treatment fails to bring positive results.

Although this treatment allows quick healing of the bleeding site, it is associated with many side effects and contraindications, which makes it unacceptable in current healthcare facilities. Firstly, the method involves applying hot equipment directly at the bleeding site. This can damage not only the blood flow but also the skin alignment resulting in total damage to the patient's skin (3). Secondly, placing a hot object on the body directly causes severe pain to the patient that can worsen their state instead of inducing healing. In that case, this method cannot be used to treat young children or people who fear pain. Lastly, heat from the hot object may cause damage to organs near the bleeding site. Apart from the possible side effects of Agni karma mentioned above, the method is associated with many contraindications, which makes it unacceptable in hospitals. For instance, it cannot be used in patients with thin skin since the heat may affect the bone (3). Also, it is not applicable in patients with bleeding disorders and those who are pregnant. Lastly, it cannot treat internal bleeding.

Following this, medical agencies have rejected Agni karma as a therapeutic procedure as it could not be of much importance due to its side effects and contraindications. Since endometriosis affects organs in the pelvis, using Agni karma will only cause injury to the anterior parts of the skin and will never result in alleviating symptoms, neither curing the disease such as our patient. In this case, legal surgical procedures are required to treat endometriosis. The two major types of surgery involving organs in the uterus include conservative surgery and hysterectomy. Conservative surgery consists of procedures such as laparoscopy, which is the use of a laparoscope to examine and remove endometrial tissues in the uterus (4). Hysterectomy involves surgical removal of the whole uterus in case of severe endometriosis. Unlike Agni karma, these procedures are done under a specific procedure. The patient has to sign a written consent first to ascertain that she is ready for any outcomes. Also, the procedures are done under general anesthesia, meaning that the patient will not go through pain (5). Lastly, surgical procedures are performed by qualified physicians who are assisted by other healthcare professionals; hence, mistakes made can be corrected before they lead to adverse outcomes.

Following the discussion above, it is clear that endometriosis can be better treated surgically in legal healthcare facilities. Such facilities are wellequipped with surgical equipment necessary for removing endometrial tissues. On the contrary, therapeutic heat burns are carried out by traditional physicians who do not understand the anatomy and physiology of the body. As such, they are likely to make mistakes that can damage other organs of the body. Also, therapeutic heat burns have many side effects and contraindications, making them unfavorable for use in legal healthcare facilities. To avoid unnecessary body damages, only qualified healthcare physicians should diagnose and treat patients with endometriosis.

\section{Conclusion}

Agni karma should be avoided to prevent the constant side effects and contraindications. Patients who have endometriosis should visit legal healthcare centers where they can receive medical and surgical treatment from qualified healthcare professionals.

\section{Competing interests}

The author(s) declare no competing interest concerning the research, authorship, and publication of this article.

\section{Consent}

Written informed consent was obtained from the patient for publication of this case report and accompanying images.

\section{References}

1. Stovall DW. (2018). What Is New in Endometriosis?: Best Articles of the Past Year Obstet Gynecol. 132(4):1056-1058.

2. Nagnath SR. (2019). A Critical Appraisal of Ancient Surgical Instruments. Research \& Reviews:. Journal of Medical Science and Technology. 2(1):10-14.

3. Nakhate SR, Giri SV. (2017). Effect of Agnikarma in calcaneal spur: A case study. Ayurline: IJ-RIM. 1(02).

4. Clark NV, Dmello M, Griffith KC, Gu X, Ajao MO, Cohen SL, et al. Laparoscopic treatment of endometriosis and predictors of major complications: a retrospective cohort study. Acta Obstet Gynecol Scand. 2019.

5. Ramirez PT, Frumovitz M, Pareja R, Lopez A, Vieira M, Ribeiro $\mathrm{R}$, et al. (2018). Minimally invasive versus abdominal radical hysterectomy for cervical cancer. N Engl J Med. 379(20):1895904. 